\title{
MODELLING OF THE MAGNETIC CONFIGURATION OF CP STARS FROM POLARIMETRIC OBSERVATIONS
}

\author{
M. LANDOLFI \\ Osservatorio di Arcetri \\ I-50125 Firenze, Italia
}

\section{Introduction}

The observational quantities commonly used to study the magnetic field of CP stars - the mean field modulus and the mean longitudinal field, as well as the 'mean asymmetry of the longitudinal field' and the 'mean quadratic field' recently introduced by Mathys (1995a,b) - are based either on the Stokes parameter $I$ or on the Stokes parameter $V$. However, a complete description of polarized radiation requires the knowledge of the full Stokes vector: in other words, we should expect that useful information is also contained in linear polarization (the Stokes parameters $Q$ and $U$ ); or rather we should expect the information contained in $(Q, U)$ and in $V$ to be complementary, since linear and circular polarization are basically related to the transverse and the longitudinal component of the magnetic field, respectively.

Very few measurements of linear polarization in CP stars have been performed so far. As to the measurement of linear polarization profiles in a spectral line, the only attempt is that of Borra \& Vaughan (1976) on $\beta$ CrB.

The measurement of broad band linear polarization (BBLP), compared with the measurement of linear polarization profiles in a spectral line, is a sort of short cut. On the one hand we waste information, because in a band of some hundreds $\AA$ there are many lines with different properties whose contributions to the overall polarization $\mathrm{mix}$ up. On the other hand, we need no longer a spectrograph (usually the standard B filter is used), we only need a polarimeter set at the Cassegrain focus of a telescope. Therefore we have a large number of photons (because polarimeters are much more transparent than any spectrograph) and a small instrumental polarization (because we avoid the mirror reflections of Coudé spectrographs). Measurements of BBLP were first performed by Kemp \& Wolstencroft (1974) then by Piirola \& Tuominen (1981), on 53 Cam and HD 71866 respectively.

Over the last few years we performed systematic observations of BBLP in CP stars, using the $2 \mathrm{~m}$ telescope of the Pic du Midi Observatory (France). The complete set of measurements has been presented by Leroy (1995). We have developed a simple theory to interpret the observations (Landolfi et al. 1993; Bagnulo et al. 1995; Leroy et al. 1996); here I'll try to summarize both the relevant physical approximations and the main results of data analysis.

\section{Interpretation of the observations}

The main features of BBLP observations are the following. The fractional polarization is of some units times $10^{-4}$, with a typical error of $1 \times 10^{-4}$, and changes periodically with time. The period is generally the same as that obtained from other kinds of measurement (longitudinal field, or photometry). If the data are displayed on a $U$ vs. $Q$ plot, we get curves with typical shapes, denoted as 'polarization diagrams'.

The most natural explanation for the existence of BBLP is provided by the differential saturation mechanism, first proposed by Leroy (1962) to explain a similar phenomenon discovered by Dollfus in sunspots. According to this mechanism, the BBLP results from all the (magnetic) spectral lines contained in the passband, via the combined effect of a magnetic field and of radiative transfer. The time variation of BBLP - which occurs with the same period as other observational quantities hence presumably with the star's rotation period - is a direct consequence of rotation if we assume a

$$
679
$$


frozen magnetic configuration on the stellar surface. Because of rotation, the magnetic configuration on the visible hemisphere changes with time, and the polarization changes accordingly.

The polarization at a given time (or a given rotational phase) is obtained by integrating the Stokes parameters $Q$ and $U$ coming from a point of the surface (where a given magnetic field vector is present) over frequency and over the visible hemisphere. The fractional polarization, which is the quantity actually observed, is obtained dividing by the analogous integral of the Stokes parameter $I$.

We have tried to interpret the observations with a simplified theory, which follows from a number of drastic approximations. The main assumption is that all the lines contained in the passband are equal and unblended, i.e., we assume the polarization is produced by an 'average' line (a Zeeman triplet) with assigned characteristics. Then we assume the stellar atmosphere is chemically homogeneous and described by the Milne-Eddington model. Finally, we assume a limb-darkening law proportional to $(1-u+u \cos \theta)$, with $0 \leq u \leq 1$, which is more realistic although, strictly speaking, inconsistent with the Milne-Eddington approximations which correspond to $u=1$. Under these assumptions, the calculation of BBLP reduces to the numerical integration, over frequency and over the stellar disk, of the polarization given by the Unno-Rachkovsky expressions for an individual line (the 'average' line).

Obviously, the polarization depends crucially on the magnetic configuration at the star's surface. At first we assumed the classical dipolar, centered oblique rotator model. The configuration is then specified by the angles $i$ (between the rotation axis and the line of sight) and $\beta$ (between the magnetic axis and the rotation axis). We can expect the shape of the polarization diagrams to be strongly sensitive to the values of $i$ and $\beta$; on the contrary, their amplitude will not be very significant, because it is affected by the magnetic field strength, the number of lines contained in the passband, the parameters characterizing the 'average' line, and the limb-darkening constant $u$, and different combinations of these quantities may produce the same amplitude.

Though straightforward, the numerical calculation of the integrals of the Stokes parameters is rather time-consuming, so it is not very practical to fit the observations. A major simplification is obtained by introducing the weak magnetic field approximation. If the field is weak - in the sense that the ratio $v_{H}$ between Zeeman splitting and line Doppler width is much less than unity the expressions of the Stokes parameters can be expanded into power series of $v_{H}$. The integrals over the visible hemisphere can be evaluated analytically, and we finally get simple analytical formulae for the BBLP (Landolfi et al. 1993). The weak field approximation (which implies a field strength less than about $2 \mathrm{kG}$ ) is generally not satisfied in magnetic CP stars; however, a systematic comparison with the BBLP obtained from the numerical integration has shown that the shapes of the polarization diagrams are well reproduced by the analytical formulae, at least up to values of the polar field of the order of $15 \mathrm{kG}$ (Bagnulo et al. 1995). These shapes are found to be strongly dependent on the values of $i$ and $\beta$, which means that BBLP is especially useful for determining the magnetic geometry.

The analytical formulae can be used directly to fit the observations, by searching for the $\chi^{2}$ minimum in the parameters' space - of course we must add to the formulae the contribution of interstellar polarization, which can produce a global shift of the polarization diagram. However, if for a given star longitudinal field measurements are also available, it is convenient to minimize the overall $\chi^{2}$, built from both sets of data: this allows one to deduce the field modulus (which cannot be deduced from linear polarimetry alone) and leads to a more reliable determination of the angles $i$ and $\beta$ (Landolfi et al. 1997).

Let's see what results are found when this procedure is applied to individual stars. In some cases, observations and theory are consistent. An example is HD 24712, where both BBLP and longitudinal field measurements are well fitted by the same dipolar model, corresponding to specific values of the rotation period, the phase constant, and the $i$ and $\beta$ angles (Bagnulo et al. 1995). Examples like this one seem to confirm the basic assumption that the magnetic configuration of CP stars is purely dipolar.

But in other cases no reliable fit can be obtained. This occurs not only for stars like 49 Cam, where the line-blocking factor changes considerably with time (which suggests that abundance inhomogeneities may play a major role), but also for stars where this factor is almost constant, like $\beta \mathrm{CrB}$ : in these cases, we have therefore a strong indication that the magnetic configuration is not purely dipolar. 
To deal with this problem, we have followed two different approaches. The basic idea of Leroy has been to look for a magnetic configuration as close as possible to the dipole. Thus he developed a model where the field modulus at the star's surface is still purely dipolar, while the inclination of the lines of force is changed in a band about the magnetic equator, through an expansion into spherical harmonics, in such a way that the lines of force are still symmetrical about the magnetic equator and are still contained in their meridian planes. This model, referred to as 'modified dipole', leads in some cases to definitely better fits (Leroy et al. 1996).

At the same time, we have tried to follow a more conventional - but more general - way by assuming the field to be the superposition of a dipole and a quadrupole. However, we found that the simplest type of quadrupole (the linear quadrupole, where the two elementary dipoles lie along the same straight line) produces polarization diagrams very similar to the dipole. Thus we had to resort to the general quadrupole, where the two opposite dipoles are displaced by a vector which is not parallel to the dipole direction. Of course this model produces a rather complex magnetic configuration: in the presence of a dipole plus a general quadrupole, the configuration is specified by three directions (one for the dipole, two for the quadrupole) so that the polarization depends on four additional angles. Work on this subject is still in progress, but the first results suggest that this magnetic model could as well be adequate to explain the observations.

\section{Conclusion}

When the simple theory outlined above is applied to observational data, a composite picture comes out: some of the observed $\mathrm{CP}$ stars can be understood in terms of the classical oblique rotator model, while some cannot. However, we must bear in mind that the errors on BBLP measurements are rather large, $10 \%$ or even more. Since for several stars we are induced to give up the dipolar model and to introduce more sophisticated magnetic models, the possibility of reducing the errors would be extremely important to set more strict constraints and to discriminate between different models. The large telescopes of the new generation, able to collect more light and thus to reduce, in principle, the observational errors, could therefore give a substantial contribution to the analysis based on BBLP.

On the other hand, the possibility of collecting more light will also make easier the measurement of linear polarization profiles in single spectral lines: this will provide a safer way to use the diagnostic content of linear polarization. In fact we should not forget that the measurement of BBLP is only a short cut: it has some practical advantages but it also has the drawback of mixing the contributions of several lines, with inevitable waste of information.

\section{References}

Bagnulo, S., Landi Degl'Innocenti, E., Landolfi, M. and Leroy, J.L. (1995) Linear polarimetry of Ap stars III. A diagnostic method for the magnetic structure of rotating stars, Astron. Astrophys. 295, pp. 459-470

Borra, E.F. and Vaughan, A.H. (1976) Observations of the transverse Zeeman effect in the magnetic star Beta Coronae Borealis: Evidence for the oblique rotator model, Astrophys. J. 210, pp. L145-L147

Kemp, J.C. and Wolstencroft, R.D. (1974) The intrinsic linear polarization of 53 Camelopardalis and $\alpha^{2}$ Canum Venaticorum, Mon. Not. Roy. Astron. Soc. 166, pp. 1-18

Landolf, M., Landi Degl'Innocenti, E., Landi Degl'Innocenti, M. and Leroy, J.L. (1993) Linear polarimetry of Ap stars I. A simple canonical model, Astron. Astrophys. 272, pp. 285-298

Landolfi, M., Bagnulo, S., Landi Degl'Innocenti, M., Landi Degl'Innocenti, E. and Leroy, J.L. (1997) Constraints on the magnetic configuration of Ap stars from simple features of observed quantities, Astron. Astrophys. 322, pp. $197-201$

Leroy, J.L. (1962) Contributions a l'étude de la polarisation de la lumière solaire, Ann. Astrophys. 25, pp. 127-164

Leroy, J.L. (1995) Linear polarimetry of Ap stars V. A general catalogue of measurements, Astron. Astrophys. Suppl. 114, pp. 79-104

Leroy, J.L., Landolfi, M. and Landi Degl'Innocenti, E. (1996) Linear polarimetry of Ap stars VI. A modified dipolar model consistent with the observations, Astron. Astrophys. 311, pp. 513-522

Mathys, G. (1995a) Spectropolarimetry of magnetic stars IV. The crossover effect, Astron. Astrophys. 293, pp. 733745

Mathys, G. (1995b) Spectropolarimetry of magnetic stars V. The mean quadratic magnetic field, Astron. Astrophys. 293, pp. $746-763$

Pirola, V. and Tuominen, I. (1981) The intrinsic linear polarization of the magnetic variable HD 71866, in: CP stars of the upper main sequence, 23rd International Conference on Astrophysics, Liège, Université de Liège, pp. 283-288 